\title{
Efficient synthesis of novel functionalized pyrazolo-pyranoquinoline and tetrahydrodibenzo-[1,8]naphthyridinone derivatives
}

\author{
Morteza Shiri,* Masumeh Heydari, Vahideh Zadsirjan \\ Department of Chemistry, Alzahra University, Vanak, Tehran 1993893973, Iran, \\ Fax:+982188041344, mshiri@alzahra.ac.ir
}

\begin{abstract}
The facile and efficient synthesis of 1,4-dihydropyrazolo-pyrano-[2,3-b]quinoline derivatives from the reaction of 2-chloroquinoline-3-carbaldehydes and pyrazolones is described. Moreover, a one-pot method for the development of functionalized tetrahydrodibenzo $[b, g][1,8]$ naphthyridinone derivatives is reported by using a three-component reaction of 2-chloroquinoline 3-carbaldehydes, pyrazolone, and enaminones catalyzed by Lproline in $\mathrm{EtOH}$.
\end{abstract}

Keywords: Multicomponent reaction, 2-chloroquinoline 3-carbaldehyde, pyrazolone derivatives, enaminones, $[1,8]$ naphthyridinone, organocatalyst

\section{Introduction}

Small-ring heterocycles have obtained various applications both as valuable starting precursors in the formation of more complex structures and as useful targets for synthesis. Multicomponent reactions (MCRs) successfully combine three, or occasionally more, reactants simultaneously in a one-pot reaction that does not need the isolation of intermediate compounds. This strategy is one of the more important pathways for modern organic chemistry since it can utilize most of the component atoms of various reactant compounds to produce a target product. ${ }^{1}$ MCRs are also often selective and are easily performed under mild conditions; some were applied industrially. ${ }^{2}$ MCRs were extensively employed in organic synthesis to produce novel CC bonds. ${ }^{3,4}$

Heterocycles including pyrazole rings are important target molecules in synthetic and medicinal chemistry since the ring is a key scaffold in different biologically active products. Pyrazolone is one of the most prevalent $N$-heteroaromatic molecules incorporated into the 
structures of diverse pharmaceuticals. ${ }^{5}$ Pyrazolone is a five-membered lactam ring molecule with two nitrogen atoms, that is an active ingredient of different drugs, particularly in the class of nonsteroidal anti-inflammatory agents. 6,7

Quinoline derivatives exhibit a wide range of pharmacological properties, for example antiplasmodial,${ }^{8,9}$ cytotoxic, ${ }^{10}$ antibacterial, ${ }^{11}$ functional, ${ }^{12}$ antimalarial, ${ }^{13}$ antiproliferative, ${ }^{14}$ and anticancer properties. ${ }^{15}$ So, the formation of quinolones has received considerable attention in organic synthesis.

The construction of short and effective procedures for the synthesis of structurally complex products having biological properties from easily accessible starting compounds is a main challenge in both the pharmaceutical industry and academia.

2-Chloroquinoline-3-carbaldehydes, ${ }^{16}$ are key moieties used for the formation of diverse heterocyclic molecules. The reaction of 2-chloroquinoline-3-carbaldehydes with methylene active compounds has been widely investigated ${ }^{17}$ for the synthesis of various molecules such as pyrano[4,3-b]quinolones, ${ }^{18,19}$ pyrazole[3,4-b]quinolones, ${ }^{20}$ isoxazolo[5,4- $\left.b\right]$ quinolones, ${ }^{21}$ quinolino[3,2-f][1,2,4]triazolo[4,3-b]-[1,2,4]triazepines, ${ }^{22}$ and benzo $[g]$ naphtho $[b][1,8]$ naphthyridines. ${ }^{23} \mathrm{Fu}$ and co-workers found that the three component reaction of 2-chloroquinoline-3-carbaldehyde, 1,3-dicarbonyl compounds, and enaminones led solely to the formation of benzo[b][1,8]naphthyridine derivatives. ${ }^{24}$ After that, we reported the synthesis of novel functionalized 4H-pyrano[2,3-b]quinoline and 1,4dihydrobenzo $[b][1,8]$ naphthyridine derivatives via a one-pot reaction of 2-chloroquinoline-3carbaldehydes, 6-aminouracils and dimedone or 3-methyl-1H-pyrazol-5(4H)-one. ${ }^{25}$ In continuation of our research on heterocyclic compounds via MCRs, ${ }^{26,}{ }^{27}$ herein, we report an efficient synthesis of functionalized 1,4-dihydropyrazolo-pyrano-[2,3-b]quinoline and tetrahydrodibenzo $[b, g][1,8]$ naphthyridinone derivatives by using substituted 2-chloroquinoline3-carbaldehydes as starting compounds.

\section{Results and discussion}

We initially tested the reaction of the 2-chloroquinoline-3-carbaldehyde $1 \mathbf{a}^{28}$ and 3methyl-1H-pyrazol-5(4H)-one $\mathbf{2} \mathbf{a}^{29}$ (Table 1). The reaction of $\mathbf{1 a}(1 \mathrm{mmol})$ and $\mathbf{2 a}(2 \mathrm{mmol})$ was evaluated under various conditions. The effect of solvents and catalysts were considered for this transformation, and the results are shown in Table $\mathbf{1}$. 
When the reaction was carried out in EtOH in the absence of any catalyst, product 3a was formed in low yield (Table 1, entry 1 and Table 2, entry 1). Using $\mathrm{H}_{2} \mathrm{O}$ without any catalyst didn't afford any product (Table 1, entry 2). To increase the yields, we explored this reaction using various catalysts. Some bases such as $\mathrm{Cs}_{2} \mathrm{CO}_{3}, \mathrm{~K}_{2} \mathrm{CO}_{3}$, and $\mathrm{Et}_{3} \mathrm{~N}$ and Lewis acid such as $\mathrm{AlCl}_{3}$ were ineffective (Table 1, entries 5, 4, 3,8). Remarkably, L-proline was recognized as the optimal catalyst for the synthesis of $\mathbf{3 a}$ (Table 1, entry 11).

Ethanol as solvent afforded higher yields than other organic solvents such as $\mathrm{CH}_{3} \mathrm{CN}$, DMF and $\mathrm{CH}_{2} \mathrm{Cl}_{2}$ (Table 1, entry 3 vs entries 4 and 5). Therefore, ethanol was selected as the best solvent. Furthermore, we tested the quantity of L-proline needed for this reaction. The results demonstrated that $15 \mathrm{~mol} \% \mathrm{~L}$-proline at reflux in ethanol is optimal (Table 1, entries 9, 10, 11).

Table 1. Examination of various conditions for the reaction of 2-chloroquinoline-3-carbaldehyde 1a and 3-methyl-1 $H$-pyrazol-5(4H)-one 2a. ${ }^{a}$

\begin{tabular}{|c|c|c|c|}
\hline Entry & Catalyst & Solvent & Yield $(\%)^{\mathrm{a}}$ \\
\hline 1 & none & $\mathrm{EtOH}$ & 25 \\
\hline 2 & none & $\mathrm{H}_{2} \mathrm{O}$ & - \\
\hline 3 & $\mathrm{~K}_{2} \mathrm{CO}_{3}$ & $\mathrm{CH}_{3} \mathrm{CN}$ & 15 \\
\hline 4 & $\mathrm{Cs}_{2} \mathrm{CO}_{3}$ & $\mathrm{DMF}$ & 35 \\
\hline 5 & $\mathrm{AlCl}_{3}$ & $\mathrm{CH}_{2} \mathrm{Cl}$ & 10 \\
\hline 6 & $\mathrm{~K}_{2} \mathrm{CO}_{3}$ & $\mathrm{EtOH}$ & 20 \\
\hline 7 & $\mathrm{Cs}_{2} \mathrm{CO}_{3}$ & EtOH & 20 \\
\hline 8 & $\mathrm{Et}_{3} \mathrm{~N}$ & $\mathrm{EtOH}$ & 40 \\
\hline 9 & L-proline (5\%) & EtOH & 65 \\
\hline 10 & L-proline (10\%) & EtOH & 85 \\
\hline 11 & L-proline (15\%) & EtOH & 93 \\
\hline
\end{tabular}

a 2-Chloroquinoline-3-carbaldehyde $\mathbf{1 a}(1 \mathrm{mmol})$, and 3-methyl-1 $H$-pyrazol-5(4H)-one 2a (2 mmol), catalyst $(0.15 \mathrm{mmol})$, solvent $(5 \mathrm{~mL})$, reflux, $4 \mathrm{~h}$. 
The optimized reaction conditions were then evaluated for library development with 2chloroquinoline-3-carbaldehydes $1 \mathbf{a}$ and 3-methyl-1H-pyrazol-5(4H)-one 2a (Table 2). The desired functionalized 1,4-dihydropyrazolo pyrano[2,3-b]quinolines 3a-i were obtained in good yields in refluxing ethanol mediated by L-proline. As shown in Table 2, this procedure was efficient for various 2-chloroquinoline-3-carbaldehydes 1a-e bearing electron donating and electron withdrawing groups and pyrazolone derivatives such as 3-methyl-1H-pyrazol-5(4H)-one 2a, 3-phenyl-1H-pyrazol-5(4H)-one 2b and 3-(chloromethyl)-1 $H$-pyrazol-5(4H)-one 2c.

Table 2. Synthesis of novel 1,4-dihydropyrazolo-pyrano-[2,3-b]quinoline derivatives. ${ }^{\text {a }}$<smiles>[H][Y1]1ccc2cc(C=O)c(Cl)nc2c1</smiles>

1<smiles>[R]C1=NNC(=O)C1</smiles>

2

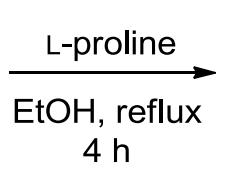

$4 \mathrm{~h}$<smiles></smiles>

3

\begin{tabular}{|c|c|c|c|}
\hline Entry & $\mathrm{R}^{1}$ & $\mathrm{R}^{2}$ & Yield 3 (\%) \\
\hline 1 & $6-\mathrm{Me}(\mathbf{1 a})$ & $\mathrm{Me}(\mathbf{2 a})$ & $\mathbf{3 a}(93)$ \\
\hline 2 & $\mathrm{H} \mathrm{(1b)}$ & $\mathrm{Me}(\mathbf{2 a})$ & $\mathbf{3 b}(88)$ \\
\hline 3 & $6-\mathrm{Me}(\mathbf{1 c})$ & $\mathrm{Me} \mathrm{(2a)}$ & $\mathbf{3 c}(93)$ \\
\hline 4 & $\mathrm{H} \mathrm{(1b)}$ & $\mathrm{Ph}(\mathbf{2 b})$ & $\mathbf{3 d}(80)$ \\
\hline 5 & $8-\mathrm{Me}(\mathbf{1 d})$ & $\mathrm{Ph}(\mathbf{2 b})$ & $\mathbf{3 e}(85)$ \\
\hline 6 & $6-\mathrm{Me} \mathrm{(1c)}$ & $\mathrm{Ph}(\mathbf{2 b})$ & $\mathbf{3 f}(82)$ \\
\hline 7 & $6-\mathrm{OMe}(\mathbf{1 a})$ & $\mathrm{Ph}(\mathbf{2 b})$ & $\mathbf{3 g}(87)$ \\
\hline 8 & $\mathrm{H} \mathrm{(1b)}$ & $\mathrm{CH}_{2} \mathrm{Cl}(\mathbf{2 c})$ & $\mathbf{3 h}(73)$ \\
\hline 9 & $6-\mathrm{Cl}(\mathbf{1 e})$ & $\mathrm{Me} \mathrm{(2a)}$ & $\mathbf{3 i}(88)$ \\
\hline
\end{tabular}

${ }^{\mathrm{a}}$ Aldehyde 1 ( $\left.1 \mathrm{mmol}\right)$ and pyrazolone derivatives 2 (2 mmol), L-proline (0.15 mmol), EtOH (5 mL), reflux, $4 \mathrm{~h}$. 
The newly synthesized products were characterized by melting point and FT-IR, ${ }^{1} \mathrm{H}-\mathrm{NMR}$, and ${ }^{13} \mathrm{C}-\mathrm{NMR}$ spectroscopy, as well as elemental analysis. Compound 3a exhibited characteristic IR stretching frequencies in the 3301 and 1614 regions for $\mathrm{NH}$ and $\mathrm{C}=\mathrm{O}$, respectively. The ${ }^{1} \mathrm{H}-\mathrm{NMR}$ spectrum showed two methyl groups as singlets at $\delta=1.89(3 \mathrm{H})$ and $1.96(3 \mathrm{H}) \mathrm{ppm}$. Two singlets at $\delta=3.84(3 \mathrm{H})$ and $\delta=5.31(1 \mathrm{H})$ ppm corresponded to the $\mathrm{OMe}$ and $\mathrm{CH}$ of the pyran ring respectively. Four aromatic protons appeared at $7.32(2 \mathrm{H}), 7.72(1 \mathrm{H})$ and $8.01(1 \mathrm{H}) \mathrm{ppm}$. Three broad singlet peak at $\delta=9.52, \delta=11.23$ and $12.01 \mathrm{ppm}$ each for one proton attributed to three NH. The ${ }^{13} \mathrm{C}-\mathrm{NMR}$ data showed four peaks in the aliphatic region at 10.1 and $10.4 \mathrm{ppm}$ for two $\mathrm{CH}_{3}$ and at $27.5 \mathrm{ppm}$ for $\mathrm{CH}$ and $55.9 \mathrm{ppm}$ for $\mathrm{CH}_{3} \mathrm{O}$ as well as other peaks at 97.8, 105.5, 105.9, 122.3, 122.7, 127.6, 128.8, 136, 138.9, 141.0, 155.2, 156.8 and 159.9 ppm corresponding to structure 3a $(\mathrm{ESI})$.

In order to expand the scope of the process, the reaction of 5-chloro-3-methyl-1-phenyl-1Hpyrazole-4-carbaldehyde $4^{30}$ and 3-methyl-1H-pyrazol-5 $(4 H)$-one $2 \mathbf{a}$ in the presence of L-proline under reflux conditions was examined to afford novel compound $\mathbf{5}$ in $95 \%$ yield (Scheme 1).

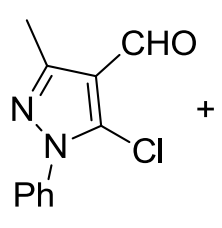

4

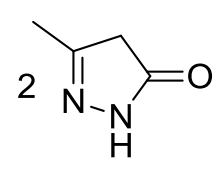

2

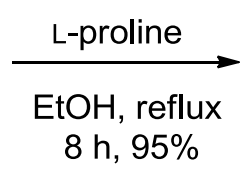

$8 \mathrm{~h}, 95 \%$

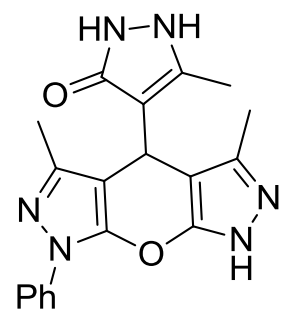

5

Scheme 1. Synthesis of novel compound 5.

Regarding this type of products, it should be mentioned that Ryabukhin et al. have studied the reaction of heteroaromatic dialkylaminoaldehydes with active methylene compounds in the presence of $\mathrm{Me}_{3} \mathrm{SiCl}^{20 \mathrm{a}}$ Under these conditions in all cases, dialkylamines appeared as a part of the target molecules. Only from the reaction of 2-(piperidin-1-yl)quinoline-3carbaldehyde with 4-(chlorophenyl)-5-methyl-2,4-dihydro-3H-pyrazol-3-one the corresponding 1,4-dihydropyrazolo-pyrano-[2,3-b]quinoline has been isolated by elimination of piperidine. ${ }^{20 a}$

In the following, we want to explore the treatment of 2-chloroquinoline-3-carbaldehyde and various active methylenes in enamine-based 3-CRs. Initially, we evaluated the threecomponent reaction of the 2-chloroquinoline-3-carbaldehyde 1b, 3-methyl-1H-pyrazol-5(4H)- 
one $\mathbf{2 a}$, and enamine $\mathbf{6} \mathbf{a}^{31}$ in the presence of L-proline; under reflux conditions the corresponding functionalized tetrahydrodibenzo[b,g][1,8]naphthyridinone derivatives 7a were produced in good yields (Table 3).

Based on these reaction conditions, we checked the reaction of different 2chloroquinoline-3-carbaldehydes 1b-d and 1f 3-phenyl-1H-pyrazol-5(4H)-one 2a and enamines 6a-c. The desired tetrahydrodibenzo[ $b, g][1,8]$ naphthyridinone derivatives $\mathbf{7 a - h}$ were obtained in good yields. As demonstrated in Table $\mathbf{3}$, this procedure was efficient with various enamines. Remarkably, the enaminones were synthesized formerly from the treatment of dimedone and aryl amines. ${ }^{31}$ Notably, phenyl groups containing either electron-withdrawing or electron-donating groups on the enaminone ring, were tolerated under the reaction conditions affording the desired compounds in good yields (56-95\%) (Table 3).

Table 3. Synthesis of diverse tetrahydrodibenzo[b,g][1,8]naphthyridinone derivatives $\mathbf{7 a - h} .^{\text {a }}$

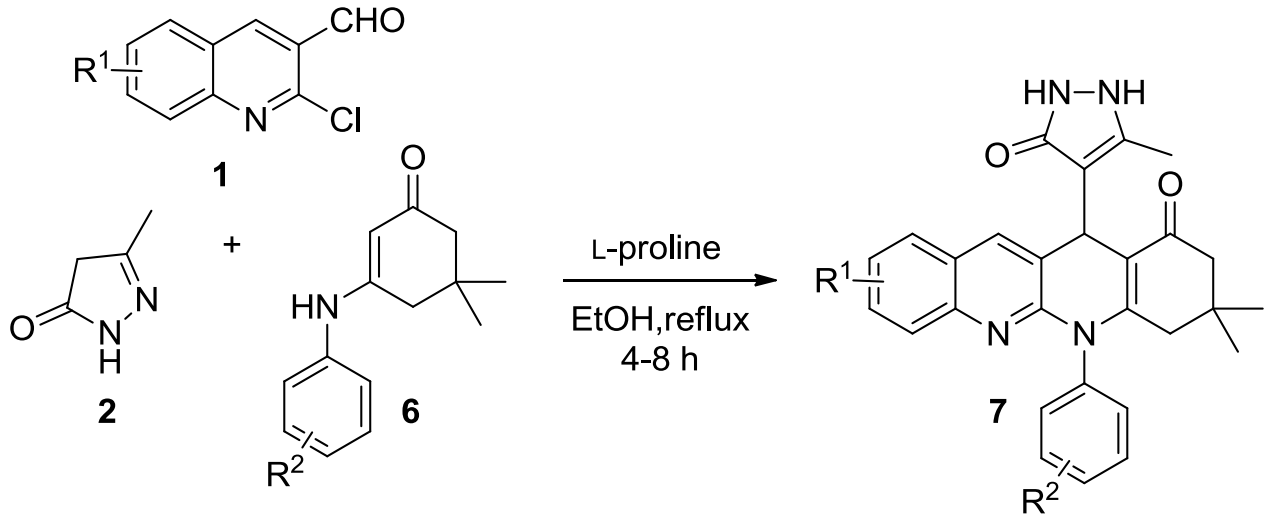

\begin{tabular}{|c|c|c|c|}
\hline Entry & $\mathrm{R}^{1}$ & $\mathrm{R}^{2}$ & Yield 7 (\%) \\
\hline 1 & $\mathrm{H} \mathrm{(1b)}$ & $4-\mathrm{OMe}(\mathbf{6 a})$ & $\mathbf{7 a}(95)$ \\
\hline 2 & $\mathrm{H} \mathrm{(1b)}$ & $4-\mathrm{Me}(\mathbf{6 b})$ & $\mathbf{7 b}(85)$ \\
\hline 3 & $\mathrm{H} \mathrm{(1b)}$ & $4-\mathrm{Cl}(\mathbf{6 c})$ & $\mathbf{7 c}(83)$ \\
\hline 4 & $6-\mathrm{Me}(\mathbf{1 c})$ & $4-\mathrm{Cl}(\mathbf{6 c})$ & $\mathbf{7 d}(89)$ \\
\hline 5 & $6-\mathrm{Br}(\mathbf{1 f})$ & $4-\mathrm{OMe}(\mathbf{6 a})$ & $\mathbf{7 e}(56)$ \\
\hline 6 & $8-\mathrm{Me}(\mathbf{1 d})$ & $4-\mathrm{Me} \mathrm{(6b)}$ & $\mathbf{7 f}(89)$ \\
\hline
\end{tabular}




\begin{tabular}{|c|c|c|c|}
\hline 7 & $8-\mathrm{Me} \mathrm{(1d)}$ & $4-\mathrm{OMe}(\mathbf{6 a})$ & $\mathbf{7 g}(94)$ \\
\hline 8 & $8-\mathrm{Me} \mathrm{(1d)}$ & $4-\mathrm{Cl}(\mathbf{6 c})$ & $\mathbf{7 h}(87)$ \\
\hline
\end{tabular}

${ }^{a}$ Aldehyde 1 ( $\left.1 \mathrm{mmol}\right)$, pyrazolone 2 ( $\left.1 \mathrm{mmol}\right)$, enamine 6 (1 mmol) L-proline (0.15 mmol), EtOH (5 mL), reflux, 4-8 h

The enaminones were synthesized formerly from the treatment of dimedone and arylamine, here we tried to evaluate the four-component reaction of 2-chloroquinoline-3carbaldehyde 1b, 3-methyl-1H-pyrazol-5(4H)-one 2a dimedome and 4-methylaniline in the presence of L-proline in EtOH under reflux conditions. But, the desired product $\mathbf{7 b}$ was provided in only $20 \%$ yield (Scheme 2).<smiles>CC1=NNC(=O)C1</smiles><smiles>Cc1ccc(N)cc1[CH+]C1(C)CC(=O)CC(=O)C1</smiles><smiles>CCO[Pb][Te]O</smiles>
$8 \mathrm{~h}$, reflux<smiles>COc1ccc(N2C3=C(C(=O)CC(C)(C)C3)C(c3c(C)[nH][nH]c3=O)c3cc4ccccc4nc32)cc1</smiles>

Scheme 2. Synthesis of compound $\mathbf{7 b}$ through four-component reaction.

A suggested mechanism based on the proposal of Fu et al. ${ }^{24}$ is shown in Scheme 3. It was proposed that L-proline mediates the production of iminum $\mathbf{A}$ in a reversible reaction with the 2chloroquinoline-3-carbaldehyde $\mathbf{1}$. The higher reactivity of the iminum ion compared with the carbonyl species could improve Knoevenagel reaction with 3-methyl-1 $H$-pyrazol-5(4H)-one 2, through intermediate $\mathbf{B}$, and after the elimination of L-proline, $\mathbf{C}$ might be provided as an intermediate. The addition of $\mathbf{C}$ to enaminone $\mathbf{6}$ then could accomplish the intermediate $\mathbf{D}$, that after intramolecular cyclization provide products 7 . 


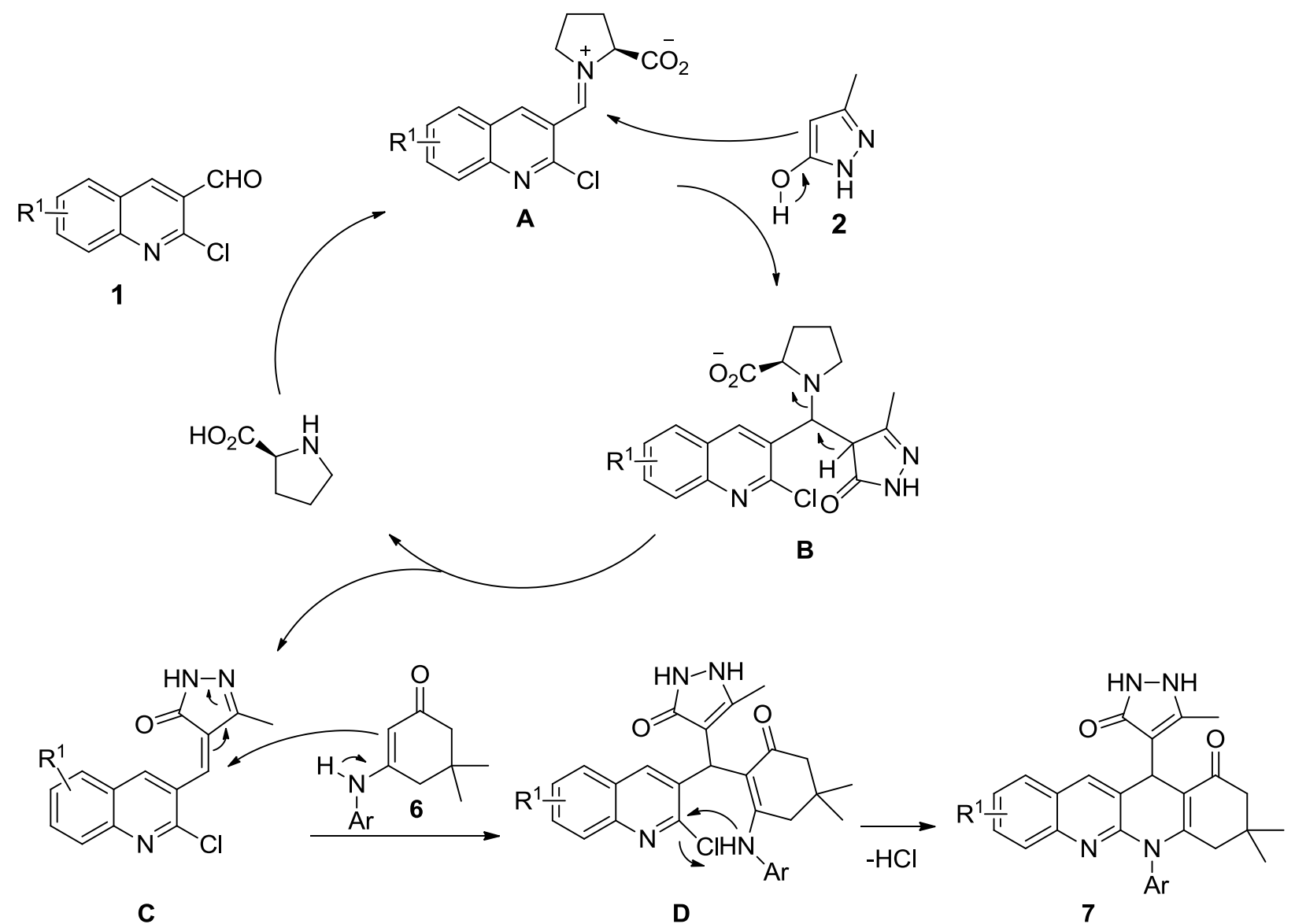

Scheme 3. Proposed mechanism for the synthesis of 7.

\section{Conclusions}

In conclusion, novel functionalized 1,4-dihydro pyrazolo pyrano[2,3-b]quinoline derivatives were synthesized. This method was accomplished via a facile and efficient reaction of 2chloroquinoline-3-carbaldehyde and pyrazolone derivatives under L-proline catalysis in EtOH under reflux conditions. Moreover, the typical one-pot, three-component reaction of 2chloroquinoline-3-carbaldehyde and 3-methyl-1H-pyrazol-5(4H)-one and enamines afforded tetrahydrodibenzo $[b, g][1,8]$ naphthyridinone derivatives in good to excellent yields. Some advantageous of this procedure are operational simplicity, good to high yields and organocatalysis reaction conditions.

\section{Experimental section}


All solvents and reagents were used as supplied from commercial sources. Melting points were taken by using a 9200 -Branread Electrothermal. ${ }^{1} \mathrm{H}-\mathrm{NMR}$ and ${ }^{13} \mathrm{C}$ NMR spectra were recorded on a Bruker 400 spectrometer. ${ }^{1} \mathrm{H}-\mathrm{NMR}$ spectra were referenced to tetramethylsilane $(0.00 \mathrm{ppm})$ using DMSO- $\mathrm{d}_{6}$-as solvent. ${ }^{13} \mathrm{C}-\mathrm{NMR}$ spectra were referenced to solvent carbons. IR spectra were recorded using an FT-IR Tensor 27. High-resolution mass data were obtained using a Bruker micro TOF-Q II ESI instrument operating at ambient temperature. A Leco CHNS, model 932 was used for elemental analysis.

\subsection{General methods}

4.2. General procedure for the synthesis of compounds 3 and 5: A dry $25 \mathrm{~mL}$ flask was charged with aldehyde $(1 \mathrm{mmol})$ and pyrazolone derivatives $(2 \mathrm{mmol}), \mathrm{L}$-proline $(0.017 \mathrm{~g}, 0.15$ mmol) and ethanol $(5 \mathrm{~mL})$. The mixture was stirred at reflux for the appropriate time. After reaction completion (TLC), the reaction mixture was cooled to room temperature. The mixture was filtered and the precipitate washed several times with EtOH to afford the product. Copies of NMR spectra for all compounds are provided in the ESI.

4.2.1. 4-(7-Methoxy-3-methyl-1,4-dihydropyrazolo[4',3':5,6]pyrano[2,3-b]quinolin-4-yl)-5methyl-1H-pyrazol-3(2H)-one (3a): $(337 \mathrm{mg}, 93 \%)$ as a white powder, m.p.: > $300{ }^{\circ} \mathrm{C}$. FT-IR $(\mathrm{KBr})\left(v_{\max }, \mathrm{cm}^{-1}\right): 3351,3048,3016,2923,1600$; Anal. calc. for $\mathrm{C}_{19} \mathrm{H}_{17} \mathrm{~N}_{5} \mathrm{O}_{3}$ : C, 62.80; H, 4.72; $\mathrm{N}, 19.27 \%$, found: C, 62.71; H, 4.49; N, 19.17\%; (400 MHz, DMSO-d $\left.d_{6}\right): \delta 1.89\left(\mathrm{~s}, 3 \mathrm{H}, \mathrm{CH}_{3}\right)$, $1.96\left(\mathrm{~s}, 3 \mathrm{H}, \mathrm{CH}_{3}\right), 3.84\left(\mathrm{~s}, 3 \mathrm{H}, \mathrm{OCH}_{3}\right), 5.31(\mathrm{~s}, 1 \mathrm{H}, \mathrm{CH}), 7.32-7.34(\mathrm{~m}, 2 \mathrm{H}, \mathrm{Ar} \mathrm{CH}), 7.74(\mathrm{~d}, J=$ $12.0 \mathrm{~Hz}, 1 \mathrm{H}, \mathrm{Ar} \mathrm{CH}$ ), 8.01 (s, 1H, Ar CH), 9.52 (s, 1H, NH), 11.23 (s, 1H, NH), 12.01 (s, 1H, $\mathrm{NH})$; (100 MHz, DMSO-d $\left.d_{6}\right): \delta 10.1,10.4,27.6$, 55.9, 97.8, 105.5, 105.9, 122.3, 122.7, 127.6, 128.8, 136.0, 138.9, 141.0, 155.2, 156.8, 159.9 .

4.2.2. 5-Methyl-4-(3-methyl-1,4-dihydropyrazolo[4',3':5,6]pyrano[2,3-b]quinolin-4-yl)-1Hpyrazol-3(2H)-one $(\mathbf{3 b})$ : $(293 \mathrm{mg}, 88 \%)$ as a white powder, m.p.: > $300{ }^{\circ} \mathrm{C}$. FT-IR $(\mathrm{KBr})\left(v_{\max }\right.$, $\mathrm{cm}^{-1}$ ): 3179, 3051, 2981, 2923, 1595; Anal. calc. for $\mathrm{C}_{18} \mathrm{H}_{15} \mathrm{~N}_{5} \mathrm{O}_{2}: \mathrm{C}, 64.86 ; \mathrm{H}, 4.54 ; \mathrm{N}, 21.01 \%$, found: C, 65.01; H, 4.43; N, 21.12; (400 MHz, DMSO-d $): \delta 2.10\left(\mathrm{~s}, 3 \mathrm{H}, \mathrm{CH}_{3}\right), 2.13(\mathrm{~s}, 3 \mathrm{H}$, $\mathrm{CH}_{3}$ ), 5.10 (s, 1H, CH), 7.36-7.40 (m, 1H, Ar CH), 7.56-7.60 (m, 1H, Ar CH), 7.70-7.75 (m, 2H, $\operatorname{Ar} \mathrm{CH}), 8.10$ (s, 1H, Ar CH), 11.50 (s, 3H, NH); (100 MHz, DMSO-d $)$ : $\delta 10.9,14.9,28.4$, 103.2, 124.4, 125.3, 126.6, 127.6, 127.9, 129.1, 136.5, 144.9, 159.9. 
4.2.3. 4-(3,7-Dimethyl-1,4-dihydropyrazolo[4',3':5,6]pyrano[2,3-b]quinolin-4-yl)-5-methyl-1Hpyrazol-3(2H)-one (3c): (323 mg, 93\%) as a white powder, m.p.: > $300{ }^{\circ} \mathrm{C}$. FT-IR $(\mathrm{KBr})\left(v_{\max }\right.$, $\mathrm{cm}^{-1}$ ): 3352, 3049, 3017, 2924, 1600; Anal. calc. for $\mathrm{C}_{19} \mathrm{H}_{17} \mathrm{~N}_{5} \mathrm{O}_{2}: \mathrm{C}, 65.69 ; \mathrm{H}, 4.93 ; \mathrm{N}, 20.16 \%$, found: $\mathrm{C}, 65.47 ; \mathrm{H}, 4.82 ; \mathrm{N}, 20.11 \%$; (400 MHz, DMSO- $\left.d_{6}\right): \delta 1.88\left(\mathrm{~s}, 3 \mathrm{H}, \mathrm{CH}_{3}\right), 1.97(\mathrm{~s}, 3 \mathrm{H}$, $\left.\mathrm{CH}_{3}\right), 2.44$ (s, 3H, $\left.\mathrm{CH}_{3}\right), 5.32(\mathrm{~s}, 1 \mathrm{H}, \mathrm{CH}), 7.53$ (d, J=8.0 Hz, 1H, $\left.\mathrm{Ar} \mathrm{CH}\right), 7.65$ (s, 1H, $\mathrm{Ar} \mathrm{CH}$ ), 7.73 (d, $J=8.0 \mathrm{~Hz}, 1 \mathrm{H}, \mathrm{Ar} \mathrm{CH}), 8.00$ (s, 1H, Ar CH), 9.52 (s, 1H, NH), 11.22 (s, 1H, NH), 12.03 (s, $1 \mathrm{H}, \mathrm{NH}) ;\left(100 \mathrm{MHz}, \mathrm{DMSO}-d_{6}\right): \delta 10.1,10.4,21.4,27.6,97.9,105.6,122.1,126.5,126.7$, 127.2, 132.4, 134.8, 135.9, 136.4, 139.3, 143.8, 156.3, 156.8, 159.4.

4.2.4. 5-Phenyl-4-(3-phenyl-1,4-dihydropyrazolo[4',3':5,6]pyrano[2,3-b]quinolin-4-yl)-1Hpyrazol-3(2H)-one (3d): $(365 \mathrm{mg}, 80 \%)$ as a white powder, m.p.: > $300^{\circ} \mathrm{C}$. FT-IR $(\mathrm{KBr})\left(v_{\max }\right.$, $\mathrm{cm}^{-1}$ ): 3352, 3049, 3016, 2920, 1601; Anal. calc. for $\mathrm{C}_{28} \mathrm{H}_{19} \mathrm{~N}_{5} \mathrm{O}_{2}: \mathrm{C}, 73.51 ; \mathrm{H}, 4.19 ; \mathrm{N}, 15.31 \%$, found: $\mathrm{C}, 73.38 ; \mathrm{H}, 4.12 ; \mathrm{N}, 15.20 \%$; (400 MHz, DMSO- $\left.d_{6}\right): \delta 5.93(\mathrm{~s}, 1 \mathrm{H}, \mathrm{CH}), 7.09-7.13(\mathrm{t}, J$ $=8.0 \mathrm{~Hz}, 2 \mathrm{H}, \mathrm{Ar} \mathrm{CH}), 7.22-7.30(\mathrm{~m}, 5 \mathrm{H}, \mathrm{Ar} \mathrm{CH}), 7.42(\mathrm{~s}, 3 \mathrm{H}, \mathrm{Ar} \mathrm{CH}), 7.47-7.51(\mathrm{~m}, 1 \mathrm{H}, \mathrm{Ar}$ $\mathrm{CH}), 7.70-7.75(\mathrm{~m}, 1 \mathrm{H}, \mathrm{ArCH}), 7.87(\mathrm{~d}, J=8.0 \mathrm{~Hz}, 1 \mathrm{H}, \mathrm{Ar} \mathrm{CH}), 7.98(\mathrm{~d}, J=8.0 \mathrm{~Hz}, 1 \mathrm{H}, \mathrm{Ar}$ $\mathrm{CH}), 8.35$ (s, 1H, Ar CH), 9.74 (s, 1H, NH), 11.67 (s, 1H, NH), 12.58 (s, 1H, NH); (100 MHz, DMSO- $\left.d_{6}\right): \delta 28.9,97.8,106.2,122.4,125.6,126.7,126.8,127.4,127.8,128.0,128.3,128.7$, $129.0,129.5,130.5,139.9,145.4,156.3,157.6,160.2$.

4.2.5. 4-(9-Methyl-3-phenyl-1,4-dihydropyrazolo[4',3':5,6]pyrano[2,3-b]quinolin-4-yl)-5phenyl-1H-pyrazol-3(2H)-one (3e): $(400 \mathrm{mg}, 85 \%)$ as a white powder, m.p.: > $300{ }^{\circ} \mathrm{C}$. FT-IR $(\mathrm{KBr})\left(v_{\max }, \mathrm{cm}^{-1}\right): 3404,3213,3059,2959,2920,1646$; Anal. calc. for $\mathrm{C}_{29} \mathrm{H}_{21} \mathrm{~N}_{5} \mathrm{O}_{2}: \mathrm{C}, 73.87 ; \mathrm{H}$, $4.49 ; \mathrm{N}, 14.85 ; \mathrm{O}, 6.79 \%$, found: $\mathrm{C}, 73.72 ; \mathrm{H}, 4.31 ; \mathrm{N}, 14.70 \% ;\left(400 \mathrm{MHz}, \mathrm{DMSO}-d_{6}\right): \delta 2.69$ (s, $\left.3 \mathrm{H}, \mathrm{CH}_{3}\right), 5.92(\mathrm{~s}, 3 \mathrm{H}, \mathrm{CH}), 7.09-7.12(\mathrm{t}, J=8.0 \mathrm{~Hz}, 2 \mathrm{H}, \mathrm{Ar} \mathrm{CH}), 7.22-7.44(\mathrm{~m}, 9 \mathrm{H}, \mathrm{Ar} \mathrm{CH})$, $7.58(\mathrm{~d}, J=8.0 \mathrm{~Hz}, 1 \mathrm{H}, \mathrm{Ar} \mathrm{CH}), 7.80(\mathrm{~d}, J=8.0,1 \mathrm{H}, \mathrm{Ar} \mathrm{CH}), 8.30$ (s, $1 \mathrm{H}, \mathrm{Ar} \mathrm{CH}), 9.72(\mathrm{~s}, 1 \mathrm{H}$, $\mathrm{NH}), 11.66(\mathrm{~s}, 1 \mathrm{H}, \mathrm{NH}), 12.56(\mathrm{~s}, 1 \mathrm{H}, \mathrm{NH}) ;\left(100 \mathrm{MHz}, \mathrm{DMSO}-d_{6}\right): \delta$ 18.0, 28.9, 97.8, 106.2, 122.0, 125.3, 125.9, 126.7, 127.8, 128.3, 128.6, 128.7, 129.0, 129.5, 130.4, 130.9, 135.0, 138.5, $140.2,144.4,155.5,157.8,160.3$.

4.2.6. 4-(7-Methyl-3-phenyl-1,4-dihydropyrazolo[4',3':5,6]pyrano[2,3-b]quinolin-4-yl)-5phenyl-1H-pyrazol-3(2H)-one (3f): (386 mg, 82\%) as a white powder, m.p.: > $300{ }^{\circ} \mathrm{C}$. FT-IR $(\mathrm{KBr})\left(v_{\max }, \mathrm{cm}^{-1}\right): 3397,3167,3058,2969,2922,1612$; Anal. calc. for $\mathrm{C}_{29} \mathrm{H}_{21} \mathrm{~N}_{5} \mathrm{O}_{2}: \mathrm{C}, 73.87 ; \mathrm{H}$, $4.49 ; \mathrm{N}, 14.85 ; \mathrm{O}, 6.79 \%$, found: C, 73.54; H, 4.31; N, 14.72\%; (400 MHz, DMSO- $d_{6}$ ): $\delta 2.46$ (s, $\left.3 \mathrm{H}, \mathrm{CH}_{3}\right), 5.89(\mathrm{~s}, 1 \mathrm{H}, \mathrm{CH}), 7.10-7.14(\mathrm{t}, J=8.0,2 \mathrm{H}, \mathrm{Ar} \mathrm{CH}), 7.22-7.30(\mathrm{~m}, 5 \mathrm{H}, \mathrm{Ar} \mathrm{CH}), 7.41$ 
(s, 3H, Ar CH), 7.54-7.57 (dd, $J=8.0, J=4.0 \mathrm{~Hz}, 1 \mathrm{H}, \mathrm{Ar} \mathrm{CH}), 7.74-7.77(\mathrm{~m}, 2 \mathrm{H}, \mathrm{Ar} \mathrm{CH}), 8.23$ (s, 1H, Ar CH), 9.74 (s, 1H, NH), 11.64 (s, 1H, NH), 12.56 (s, 1H, NH); (100 MHz, DMSO- $d_{6}$ ): $\delta$ 19.1, 28.9, 97.7, 106.2, 122.3, 126.7, 127.2, 127.8, 128.3, 128.6, 128.7, 129.0, 129.5, 131.0, 132.6, 134.9, 138.5, 139.2, 143.9, 155.8, 157.7.

4.2.7. 4-(7-Methoxy-3-phenyl-1,4-dihydropyrazolo[4',3':5,6]pyrano[2,3-b]quinolin-4-yl)-5phenyl-1H-pyrazol-3(2H)-one (3g): $(424 \mathrm{mg}, 87 \%)$ as a white powder, m.p.: > $300{ }^{\circ} \mathrm{C}$. FT-IR $(\mathrm{KBr})\left(v_{\max }, \mathrm{cm}^{-1}\right): 3405,3236,3059,2957,2920,1621$; Anal. calc. for $\mathrm{C}_{29} \mathrm{H}_{21} \mathrm{~N}_{5} \mathrm{O}_{3}: \mathrm{C}, 71.45 ; \mathrm{H}$, 4.34; N, 14.37\%, found: C, 71.32; H, 4.27; N, 14.19\%; (400 MHz, DMSO- $\left.d_{6}\right): \delta 3.86$ (s, 3H, $\left.\mathrm{OCH}_{3}\right), 5.90(\mathrm{~s}, 1 \mathrm{H}, \mathrm{CH}), 7.08-7.12(\mathrm{t}, J=8.0 \mathrm{~Hz}, 2 \mathrm{H}, \mathrm{Ar} \mathrm{CH}), 7.21-7.44(\mathrm{~m}, 10 \mathrm{H}, \mathrm{Ar} \mathrm{CH}), 7.77$ (d, $J=8.0 \mathrm{~Hz}, 1 \mathrm{H}, \mathrm{Ar} \mathrm{CH}$ ), 8.27 (s, 1H, Ar CH), 9.73 (s, 1H, NH), 11.65 (s, 1H, NH), 12.53 (s, $1 \mathrm{H}, \mathrm{NH})$; (100 MHz, DMSO- $\left.d_{6}\right): \delta 28.9,56.0,97.7,106.1,122.4,122.9,126.7,127.6,127.8$, $128.2,128.6,128.7,129.0,129.5,138.8,141.0,154.8,156.8$.

4.2.8. 5-(Chloromethyl)-4-(3-(chloromethyl)-1,4-dihydropyrazolo[4',3':5,6]pyrano[2,3b]quinolin-4-yl)-1H-pyrazol-3(2H)-one (3h): (293 mg, 73\%) as a white powder, m.p.: > $300{ }^{\circ} \mathrm{C}$. FT-IR $(\mathrm{KBr})\left(v_{\max }, \mathrm{cm}^{-1}\right): 3352,3050,3017,2923$, 1601; Anal. calc. for $\mathrm{C}_{18} \mathrm{H}_{13} \mathrm{Cl}_{2} \mathrm{~N}_{5} \mathrm{O}_{2}$ : C, 53.75 ; H, 3.26; N, $17.41 \%$, found: C, 53.54; H, 3.18; N, $17.22 \%$; (400 MHz, DMSO- $\left.d_{6}\right): \delta 1.91$ (s, 2H, $\mathrm{CH}_{2} \mathrm{Cl}$ ), 1.97 (s, 2H, CH $2 \mathrm{Cl}$ ), 5.34 (s, $\left.1 \mathrm{H}, \mathrm{CH}\right), 7.45-7.49$ (m, $\left.1 \mathrm{H}, \mathrm{Ar} \mathrm{CH}\right)$, 7.67-7.75 (m, 1H, Ar CH), 7.83 (d, $J=8.0 \mathrm{~Hz}, 1 \mathrm{H}, \mathrm{Ar} \mathrm{CH}), 7.93$ (d, $J=8.0 \mathrm{~Hz}, 1 \mathrm{H}, \mathrm{Ar} \mathrm{CH}), 8.12(\mathrm{~s}, 1 \mathrm{H}, \mathrm{Ar}$ $\mathrm{CH}), 9.66(\mathrm{~s}, 1 \mathrm{H}, \mathrm{NH}), 11.27(\mathrm{~s}, 1 \mathrm{H}, \mathrm{NH}), 11.03(\mathrm{~s}, 1 \mathrm{H}, \mathrm{NH})$; $\left(100 \mathrm{MHz}, \mathrm{DMSO}-d_{6}\right): \delta 10.5$, 14.9, 27.6, 97.9, 105.6, 122.3, 124.4, 125.3, 125.5, 126.6, 126.8, 127.4, 127.8, 129.1, 130.3, $136.1,136.5,140.0,145.4,156.8$.

4.2.9. 4-(7-Chloro-3-methyl-1,4-dihydropyrazolo[4',3':5,6]pyrano[2,3-b]quinolin-4-yl)-5methyl-1H-pyrazol-3(2H)-one (3i): $(323 \mathrm{mg}, 88 \%)$ as a white powder, m.p.: > $300{ }^{\circ} \mathrm{C}$. FT-IR $(\mathrm{KBr})\left(v_{\max }, \mathrm{cm}^{-1}\right): 3397,3055,2969,2923,1612$; Anal. calc. for $\mathrm{C}_{18} \mathrm{H}_{14} \mathrm{ClN}_{5} \mathrm{O}_{2}: \mathrm{C}, 58.78 ; \mathrm{H}$, 3.84; N, 19.04\%, found: C, 58.53; H, 3.71; N, 19.11\%; (400 MHz, DMSO- $\left.d_{6}\right): \delta 1.93(\mathrm{~s}, 1 \mathrm{H}$, $\mathrm{CH}_{3}$ ), 1.97 (s, H, $\mathrm{CH}_{3}$ ), 5.34 (s, 1H, CH), 7.70 (dd, $\left.J=8.0,4.0 \mathrm{~Hz}, 1 \mathrm{H}, \mathrm{Ar} \mathrm{CH}\right), 7.85$ (d, J= 8.0 $\mathrm{Hz}, 1 \mathrm{H}, \mathrm{Ar} \mathrm{CH}), 8.08$ (d, J=4.0 Hz, 1H, Ar CH), 8.13 (s, 1H, Ar CH), 9.68 (s, 1H, NH), 11.20 (s, 1H, NH), 12.06 (s, $1 \mathrm{H}, \mathrm{NH})$; (100 MHz, DMSO- $\left.d_{6}\right): \delta 10.1,10.5,27.7,97.8,105.4,123.6$, 126.5, 127.5, 129.5, 129.7, 130.8, 136.2, 139.4, 143.9, 156.6, 157.2.

4.2.10. 4-(3,5-Dimethyl-1-phenyl-4,7-dihydro-1H-pyrano[2,3-c:6,5-c']dipyrazol-4-yl)-5-methyl1H-pyrazol-3(2H)-one (5): $(344 \mathrm{mg}, 95 \%)$ as a white powder, m.p.: > $300^{\circ} \mathrm{C}$. FT-IR (KBr) $\left(v_{\max }\right.$, 
$\mathrm{cm}^{-1}$ ): 3390, 3058, 2924, 1623; Anal. calc. for $\mathrm{C}_{19} \mathrm{H}_{18} \mathrm{~N}_{6} \mathrm{O}_{2}: \mathrm{C}, 62.97 ; \mathrm{H}, 5.01 ; \mathrm{N}, 23.19 \%$, found: C, 62.83; H, 5.12; N, 23.25\%; (400 MHz, DMSO- $\left.d_{6}\right): \delta 1.77$ (s, 3H, $\left.\mathrm{CH}_{3}\right), 1.94$ (s, 3H, $\mathrm{CH}_{3}$ ), 1.96 (s, 3H, $\left.\mathrm{CH}_{3}\right), 4.93$ (s, 1H, CH), 7.31-7.35 (m, 1H, $\left.\mathrm{Ar} \mathrm{CH}\right), 7.51-7.56(\mathrm{~m}, 2 \mathrm{H}, \mathrm{Ar} \mathrm{CH}), 7.74-$ 7.77 (m, 2H, Ar CH), 9.56 (s, 1H, NH), 11.13 (s, 1H, NH), 12.17 (s, 1H, NH); (100 MHz, DMSO- $\left.d_{6}\right): \delta 10.1,10.4,13.0,31.2,99.7,100.1,102.8,120.6,126.4,129.8,136.7,138.5,146.7$, 147.6, 157.3, 159.4, 207.0.

4.3. General procedure for the synthesis of compounds 7: A dry $25 \mathrm{~mL}$ flask was charged with aldehyde (1.5 mmol), 3-methyl-1H-pyrazol-5(4H)-one (1 mmol), enaminone ( $1 \mathrm{mmol})$, L-proline $(0.017 \mathrm{~g}, 0.15 \mathrm{mmol})$ and ethanol $(5 \mathrm{~mL})$. The mixture was stirred at reflux for the appropriate time. After reaction completion (TLC), the reaction mixture was cooled to room temperature. The mixture was filtered and the precipitate washed with $\mathrm{EtOH}$ to afford the product. Copies of NMR spectra for all compounds and HR mass spectra for some compounds are provided in the ESI.

4.3.1. 5-(4-Methoxyphenyl)-3,3-dimethyl-12-(5-methyl-3-oxo-2,3-dihydro-1H-pyrazol-4-yl)3,4,5,12-tetrahydrodibenzo[b,g][1,8]naphthyridin-1(2H)-one (7a): (456 mg, 95\%) as a white powder, m.p.: $295-297^{\circ} \mathrm{C}$. FT-IR $(\mathrm{KBr})\left(v_{\max }, \mathrm{cm}^{-1}\right): 3301,3057,3004,2958,2932,1591$; $(400$ MHz, DMSO- $d_{6}$ ): $\delta 0.86$ (s, 3H, $\mathrm{CH}_{3}$ ), $0.92\left(\mathrm{~s}, 3 \mathrm{H}, \mathrm{CH}_{3}\right), 1.97\left(\mathrm{~d}, J=16.0 \mathrm{~Hz}, 1 \mathrm{H}, \mathrm{CH}_{2}\right), 2.03$ (d, $\left.J=16.0 \mathrm{~Hz}, 1 \mathrm{H}, \mathrm{CH}_{2}\right), 2.19$ (d, $\left.J=8.0 \mathrm{~Hz}, 1 \mathrm{H}, \mathrm{CH}_{2}\right), 2.23\left(\mathrm{~d}, J=8.0 \mathrm{~Hz}, 1 \mathrm{H}, \mathrm{CH}_{2}\right), 2.36$ (s, $\left.3 \mathrm{H}, \mathrm{CH}_{3}\right), 3.88\left(\mathrm{~s}, 3 \mathrm{H}, \mathrm{OCH}_{3}\right), 5.20(\mathrm{~s}, 1 \mathrm{H}, \mathrm{CH}), 7.12(\mathrm{~d}, J=8.0 \mathrm{~Hz}, 2 \mathrm{H}, \mathrm{Ar} \mathrm{CH}), 7.30-7.34(\mathrm{~m}$, $3 \mathrm{H}, \mathrm{Ar} \mathrm{CH}), 7.38(\mathrm{~d}, J=8.0 \mathrm{~Hz}, 1 \mathrm{H}, \mathrm{Ar} \mathrm{CH}), 7.46-7.50$ (m, $1 \mathrm{H}, \mathrm{Ar} \mathrm{CH}), 7.74-7.77$ (dd, $J=8.0$, $4.0 \mathrm{~Hz}, 1 \mathrm{H}, \mathrm{Ar} \mathrm{CH}), 8.00$ (s, 1H, Ar CH), 9.45 (s, 1H, NH), 10.98 (s, 1H, NH); (100 MHz, DMSO- $\left.d_{6}\right): \delta 10.3,26.5,28.7,29.3,32.0,41.6,49.6,55.3,108.3,108.7,114.4,123.7,124.1$, 126.2, 126.9, 127.2, 128.9, 132.3, 135.2, 136.6, 144.9, 151.1, 152.8, 158.5, 159.4, 194.2; HRMS (ESI, TOF): calcd for $\mathrm{C}_{29} \mathrm{H}_{28} \mathrm{~N}_{4} \mathrm{O}_{3} \mathrm{Na}$ : 503.2059, Found: 503.2051.

4.3.2. 3,3-Dimethyl-12-(5-methyl-3-oxo-2,3-dihydro-1H-pyrazol-4-yl)-5-(p-tolyl)-3,4,5,12tetrahydrodibenzo[b,g][1,8]naphthyridin-1(2H)-one (7b): $(394 \mathrm{mg}, 85 \%)$ as a white powder, m.p.: 297-299 ${ }^{\circ} \mathrm{C}$. FT-IR (KBr) $\left(v_{\max }, \mathrm{cm}^{-1}\right)$ : 3306, 3032, 2956, 2930, 1591; Anal. calc. for $\mathrm{C}_{29} \mathrm{H}_{28} \mathrm{~N}_{4} \mathrm{O}_{2}$ : C, 74.98; $\mathrm{H}, 6.08 ; \mathrm{N}, 12.06 ; \mathrm{O}, 6.89 \%$, found: $\mathrm{C}, 74.79 ; \mathrm{H}, 6.13 ; \mathrm{N}, 12.15 \%$; 400 MHz, DMSO- $\left.d_{6}\right): \delta 0.86\left(\mathrm{~s}, 3 \mathrm{H}, \mathrm{CH}_{3}\right), 0.92\left(\mathrm{~s}, 3 \mathrm{H}, \mathrm{CH}_{3}\right), 1.95\left(\mathrm{~d}, J=16.0 \mathrm{~Hz}, 1 \mathrm{H}, \mathrm{CH}_{2}\right), 2.03$ 
$\left(\mathrm{d}, J=16.0 \mathrm{~Hz}, 1 \mathrm{H}, \mathrm{CH}_{2}\right), 2.20\left(\mathrm{~d}, J=4.0 \mathrm{~Hz}, 1 \mathrm{H}, \mathrm{CH}_{2}\right), 2.24\left(\mathrm{~d}, J=4.0 \mathrm{~Hz}, 1 \mathrm{H}, \mathrm{CH}_{2}\right), 2.26(\mathrm{~s}$, $\left.3 \mathrm{H}, \mathrm{CH}_{3}\right), 2.36\left(\mathrm{~s}, 3 \mathrm{H}, \mathrm{CH}_{3}\right), 5.20(\mathrm{~s}, 1 \mathrm{H}, \mathrm{CH}), 7.28-7.34(\mathrm{~m}, 3 \mathrm{H}, \mathrm{Ar} \mathrm{CH}), 7.36-7.40$ (m, $2 \mathrm{H}, \mathrm{Ar}$ $\mathrm{CH}), 7.46-7.50(\mathrm{~m}, 1 \mathrm{H}, \mathrm{Ar} \mathrm{CH}), 7.76$ (d, J=8.0 Hz, 1H, Ar CH), 8.00 (s, 1H, Ar CH) , 9.31 (s, $1 \mathrm{H}, \mathrm{NH}), 11.02$ (s, 1H, NH); (100 MHz, DMSO-d $): \delta$ 10.7, 21.4, 27.0, 29.1, 29.7, 32.5, 42.1, 50.1, 108.8, 109.2, 124.2, 124.6, 126.6, 127.2, 127.6, 129.4, 130.3, 135.7, 137.1, 137.7, 145.4, $151.4,153.0,159.8,194.7$.

4.3.3. 5-(4-Chlorophenyl)-3,3-dimethyl-12-(5-methyl-3-oxo-2,3-dihydro-1H-pyrazol-4-yl)3,4,5,12-tetrahydrodibenzo[b,g][1,8]naphthyridin-1(2H)-one (7c): (402 mg, 83\%) as a white powder, m.p.: 297-299 ${ }^{\circ} \mathrm{C}$. FT-IR (KBr) $\left(v_{\max } \mathrm{cm}^{-1}\right): 3294,3057,2957,2930$, 1588; (400 MHz, DMSO-d $\left.)_{6}\right): \delta 0.87\left(\mathrm{~s}, 3 \mathrm{H}, \mathrm{CH}_{3}\right), 0.94\left(\mathrm{~s}, 3 \mathrm{H}, \mathrm{CH}_{3}\right), 1.95\left(\mathrm{~d}, J=16.0 \mathrm{~Hz}, 1 \mathrm{H}, \mathrm{CH}_{2}\right), 2.05(\mathrm{~d}, J=$ $\left.16.0 \mathrm{~Hz}, 1 \mathrm{H}, \mathrm{CH}_{2}\right), 2.22\left(\mathrm{~d}, J=16.0 \mathrm{~Hz}, 2 \mathrm{H}, \mathrm{CH}_{2}\right), 2.38$ (s, 3H, $\left.\mathrm{CH}_{3}\right), 5.20$ (s, 1H, CH), 7.317.35 (m, 1H, Ar CH), 7.39-7.51 (m, 4H, Ar CH), 7.67 (d, J=8.0 Hz, 2H, Ar CH), 7.77 (d, $J=$ $8.0 \mathrm{~Hz}, 1 \mathrm{H}, \mathrm{Ar} \mathrm{CH}), 8.03$ (s, 1H, Ar CH), 9.37 (s, 1H, NH), 11.02 (s, 1H, NH); (100 MHz, DMSO- $\left.d_{6}\right): \delta 10.2,26.4,28.6,29.2,32.1,41.5,49.6,108.2,109.1,123.5,124.2,126.2,127.0$, 127.1, 129.0, 129.3, 132.5, 135.3, 136.7, 138.7, 144.7, 150.7, 151.9, 159.4, 194.2; HRMS (ESI, TOF): calcd for $\mathrm{C}_{28} \mathrm{H}_{25} \mathrm{ClN}_{4} \mathrm{O}_{2} \mathrm{Na}$ : 507.1564, Found: 507.1557.

4.3.4. 5-(4-chlorophenyl)-3,3,9-trimethyl-12-(5-methyl-3-oxo-2,3-dihydro-1H-pyrazol-4-yl)3,4,5,12-tetrahydrodibenzo[b,g][1,8]naphthyridin-1(2H)-one (7d): (444 mg, 89\%) as a white powder, m.p.: > $300{ }^{\circ} \mathrm{C}$. FT-IR $(\mathrm{KBr})\left(v_{\max }, \mathrm{cm}^{-1}\right): 3302,3059$, 3022, 2955, 2928, 1587; Anal. calc. for $\mathrm{C}_{29} \mathrm{H}_{27} \mathrm{ClN}_{4} \mathrm{O}_{2}: \mathrm{C}, 69.80 ; \mathrm{H}, 5.45 ; \mathrm{N}, 11.23 \%$, found: $\mathrm{C}, 67.01 ; \mathrm{H}, 4.52 ; \mathrm{N}, 11.27 \%$; (400 MHz, DMSO- $\left.d_{6}\right): \delta 0.87\left(\mathrm{~s}, 3 \mathrm{H}, \mathrm{CH}_{3}\right), 0.94\left(\mathrm{~s}, 3 \mathrm{H}, \mathrm{CH}_{3}\right), 1.94\left(\mathrm{~d}, J=16.0 \mathrm{~Hz}, 1 \mathrm{H}, \mathrm{CH}_{2}\right)$, $2.04\left(\mathrm{~d}, J=16.0 \mathrm{~Hz}, 1 \mathrm{H}, \mathrm{CH}_{2}\right), 2.21\left(\mathrm{~d}, J=16.0 \mathrm{~Hz}, 2 \mathrm{H}, \mathrm{CH}_{2}\right), 2.36\left(\mathrm{~s}, 3 \mathrm{H}, \mathrm{CH}_{3}\right), 2.39$ (s, 3H, $\left.\mathrm{CH}_{3}\right), 5.19(\mathrm{~s}, 1 \mathrm{H}, \mathrm{CH}), 7.30-7.35(\mathrm{~m}, 2 \mathrm{H}, \mathrm{Ar} \mathrm{CH}), 7.46$ (d, J = 8.0 Hz, 2H, Ar CH), $7.54(\mathrm{~s}, 1 \mathrm{H}$, $\operatorname{Ar} \mathrm{CH}), 7.66(\mathrm{~d}, J=8.0 \mathrm{~Hz}, 2 \mathrm{H}, \operatorname{Ar} \mathrm{CH}), 7.93$ (s, 1H, Ar CH), 9.35 (s, 1H, NH), 11.00 (s, 1H, NH); (100 MHz, DMSO- $\left.d_{6}\right): \delta$ 10.7, 21.4, 26.9, 29.1, 29.7, 32.5, 42.1, 50.1, 108.6, 109.3, 123.9, 126.3, 126.6, 127.5, 129.8, 131.6, 133.0, 133.9, 136.6, 139.2, 143.7, 150.7, 152.5, 194.7 .

4.3.5. 9-Bromo-5-(4-methoxyphenyl)-3,3-dimethyl-12-(5-methyl-3-oxo-2,3-dihydro-1H-pyrazol4-yl)-3,4,5,12-tetrahydrodibenzo[b,g][1,8]naphthyridin-1(2H)-one (7e): (313 $\mathrm{mg}, 56 \%)$ as a white powder, m.p.: > $300{ }^{\circ} \mathrm{C}$. FT-IR $(\mathrm{KBr})\left(v_{\max }, \mathrm{cm}^{-1}\right): 3269,2954,1614$; $(400 \mathrm{MHz}$, DMSO$\left.d_{6}\right): \delta 0.84\left(\mathrm{~s}, 3 \mathrm{H}, \mathrm{CH}_{3}\right), 0.90\left(\mathrm{~s}, 3 \mathrm{H}, \mathrm{CH}_{3}\right), 1.91\left(\mathrm{~d}, J=8.0 \mathrm{~Hz}, 1 \mathrm{H}, \mathrm{CH}_{2}\right), 1.96(\mathrm{~d}, J=8.0 \mathrm{~Hz}$, 
$\left.1 \mathrm{H}, \mathrm{CH}_{2}\right), 2.17\left(\mathrm{~d}, J=8.0 \mathrm{~Hz}, 1 \mathrm{H}, \mathrm{CH}_{2}\right), 2.21\left(\mathrm{~d}, J=8.0 \mathrm{~Hz}, 1 \mathrm{H}, \mathrm{CH}_{2}\right), 2.35\left(\mathrm{~s}, 3 \mathrm{H}, \mathrm{CH}_{3}\right), 3.86$ $\left(\mathrm{s}, 3 \mathrm{H}, \mathrm{OCH}_{3}\right), 5.16(\mathrm{~s}, 1 \mathrm{H}, \mathrm{CH}), 7.09$ (d, 2H, J=8.0 Hz, Ar CH), 7.31 (d, 3H, J = 4.0 Hz, Ar $\mathrm{CH}), 7.56$ (d, 2H, J=4.0 Hz, Ar CH), 7.90 (d, 2H, J=16.0 Hz, Ar CH), 9.30 (s, 1H, NH), 10.96 (s, 1H, NH); (100 MHz, DMSO-d $\left.d_{6}\right): \delta 10.2,26.5,28.7,29.3,32.0,41.6,49.6,55.3,108.1,108.9$, $114.4,116.5,117.6,123.2,124.9,127.5,128.9,129.1,129.3,131.8,132.1,132.8,135.8,143.6$, 151.5, 152.7, 158.6, 194.3; HRMS (ESI, TOF): calcd for $\mathrm{C}_{29} \mathrm{H}_{27} \mathrm{BrN}_{4} \mathrm{O}_{3} \mathrm{Na}$ : 581.1164, Found: 581.1183.

4.3.6. 3,3,7-Trimethyl-12-(5-methyl-3-oxo-2,3-dihydro-1H-pyrazol-4-yl)-5-(p-tolyl)-3,4,5,12tetrahydrodibenzo[b,g][1,8]naphthyridin-1(2H)-one (7f): (425 mg, 89\%) as a white powder, m.p.: > $300{ }^{\circ} \mathrm{C}$. FT-IR (KBr) $\left(v_{\max }, \mathrm{cm}^{-1}\right): 3303$, 3027, 2955, 2926, 1594; (400 MHz, DMSO- $\left.d_{6}\right)$ : $\delta 0.88\left(\mathrm{~s}, 3 \mathrm{H}, \mathrm{CH}_{3}\right), 0.93\left(\mathrm{~s}, 3 \mathrm{H}, \mathrm{CH}_{3}\right), 2.05\left(\mathrm{~d}, J=16.0 \mathrm{~Hz}, 2 \mathrm{H}, \mathrm{CH}_{2}\right), 2.14\left(\mathrm{~s}, 1 \mathrm{H}, \mathrm{CH}_{3}\right), 2.21$ $\left(\mathrm{d}, J=16.0 \mathrm{~Hz}, 1 \mathrm{H}, \mathrm{CH}_{2}\right), 2.36\left(\mathrm{~s}, 3 \mathrm{H}, \mathrm{CH}_{3}\right), 2.46\left(\mathrm{~s}, 3 \mathrm{H}, \mathrm{CH}_{3}\right), 5.20(\mathrm{~s}, 1 \mathrm{H}, \mathrm{CH}), 7.19-7.23(\mathrm{t}, J$ $=8.0 \mathrm{~Hz}, 1 \mathrm{H}, \operatorname{Ar} \mathrm{CH}), 7.30-7.35(\mathrm{~m}, 3 \mathrm{H}, \mathrm{Ar} \mathrm{CH}), 7.39(\mathrm{~d}, J=8.0 \mathrm{~Hz}, 2 \mathrm{H}, \mathrm{Ar} \mathrm{CH}), 7.58(\mathrm{~d}, J=$ $8.0 \mathrm{~Hz}, 1 \mathrm{H}, \mathrm{Ar} \mathrm{CH}), 7.96$ (s, 1H, Ar CH), 9.29 (s, 1H, NH), 10.99 (s, 1H, NH); (100 MHz, DMSO- $\left.d_{6}\right): \delta 10.2,16.5,20.9,26.6,28.5,29.1,32.1,41.6,49.7,108.4,108.6,123.3,123.9$, 124.7, 125.9, 128.8, 134.5, 136.7, 137.0, 137.2, 143.7, 149.9, 152.2, 194.2; HRMS (ESI, TOF): calcd for $\mathrm{C}_{30} \mathrm{H}_{30} \mathrm{~N}_{4} \mathrm{O}_{2} \mathrm{Na}$ : 501.2266, Found: 501.2271.

4.3.7. 5-(4-Methoxyphenyl)-3,3,7-trimethyl-12-(5-methyl-3-oxo-2,3-dihydro-1H-pyrazol-4-yl)3,4,5,12-tetrahydrodibenzo[b,g][1,8]naphthyridin-1(2H)-one (7g): (465 $\mathrm{mg}, 94 \%)$ as a white powder, m.p.: 294-296 ${ }^{\circ} \mathrm{C}$. FT-IR (KBr) $\left(v_{\max }, \mathrm{cm}^{-1}\right): 3301,2955,2929$, 1593; (400 MHz, DMSO-d $\left.)_{6}\right): \delta .89$ (s, 3H, $\left.\mathrm{CH}_{3}\right), 0.94\left(\mathrm{~s}, 3 \mathrm{H}, \mathrm{CH}_{3}\right), 2.05$ (d, $\left.J=12.0 \mathrm{~Hz}, 1 \mathrm{H}, \mathrm{CH}_{2}\right), 2.09(\mathrm{~d}, J=$ $\left.12.0 \mathrm{~Hz}, 1 \mathrm{H}, \mathrm{CH}_{2}\right), 2.16\left(\mathrm{~s}, 1 \mathrm{H}, \mathrm{CH}_{3}\right), 2.18\left(\mathrm{~d}, J=16.0 \mathrm{~Hz}, 1 \mathrm{H}, \mathrm{CH}_{2}\right), 2.26$ (d, J = $16.0 \mathrm{~Hz}, 1 \mathrm{H}$, $\left.\mathrm{CH}_{2}\right), 2.35\left(\mathrm{~s}, 3 \mathrm{H}, \mathrm{CH}_{3}\right), 3.88\left(\mathrm{~s}, 3 \mathrm{H}, \mathrm{OCH}_{3}\right), 5.20(\mathrm{~s}, 1 \mathrm{H}, \mathrm{CH}), 7.12(\mathrm{~d}, J=8.0,2 \mathrm{H}, \mathrm{Ar} \mathrm{CH})$, 7.19-7.23 (t, $J=8.0 \mathrm{~Hz}, 1 \mathrm{H}, \mathrm{Ar} \mathrm{CH}), 7.35$ (d, $J=8.0 \mathrm{~Hz}, 3 \mathrm{H}, \mathrm{Ar} \mathrm{CH}), 7.58$ (d, $J=8.0 \mathrm{~Hz}, 1 \mathrm{H}$, $\operatorname{Ar} \mathrm{CH}), 7.96$ (s, 1H, Ar CH), $9.30(\mathrm{~s}, 1 \mathrm{H}, \mathrm{NH}), 10.98$ (s, 1H, NH); (100 MHz, DMSO- $\left.d_{6}\right): \delta$ 10.2, 16.6, 26.6, 28.5, 29.2, 32.0, 41.6, 49.7, 55.3, 108.4, 108.6, 114.1, 123.3, 123.9, 124.7, 125.9, 128.8, 132.4, 134.5, 136.7, 143.7, 150.0, 152.8, 158.4, 194.2; HRMS (ESI, TOF): calcd for $\mathrm{C}_{30} \mathrm{H}_{30} \mathrm{~N}_{4} \mathrm{O}_{3} \mathrm{Na}$ : 517.2216, Found: 517.2231. 
4.3.8. 5-(4-Chlorophenyl)-3,3,7-trimethyl-12-(5-methyl-3-oxo-2,3-dihydro-1H-pyrazol-4-yl)3,4,5,12-tetrahydrodibenzo[b,g][1,8]naphthyridin-1(2H)-one (7h): (434 $\mathrm{mg}, 87 \%)$ as a white powder, m.p.: > $300{ }^{\circ} \mathrm{C}$. FT-IR (KBr) $\left(v_{\max }, \mathrm{cm}^{-1}\right): 3296,3026,2958,2931,1595$; $(400 \mathrm{MHz}$, DMSO- $\left.d_{6}\right): \delta 0.89$ (s, 3H, CH $\left.\mathrm{CH}_{3}\right), 0.95\left(\mathrm{~s}, 3 \mathrm{H}, \mathrm{CH}_{3}\right), 2.03\left(\mathrm{~d}, J=8.0 \mathrm{~Hz}, 1 \mathrm{H}, \mathrm{CH}_{2}\right), 2.07(\mathrm{~d}, J=$ $\left.8.0 \mathrm{~Hz}, 1 \mathrm{H}, \mathrm{CH}_{2}\right), 2.16$ (s, 3H, $\left.\mathrm{CH}_{3}\right), 2.22\left(\mathrm{~d}, J=16.0 \mathrm{~Hz}, 1 \mathrm{H}, \mathrm{CH}_{2}\right), 2.28(\mathrm{~d}, J=16.0 \mathrm{~Hz}, 1 \mathrm{H}$, $\mathrm{CH}_{2}$ ), 2.38 (s, 3H, $\mathrm{CH}_{3}$ ), 5.20 (s, 1H, CH), 7.20-7.24 (t, $\left.J=8.0 \mathrm{~Hz}, 1 \mathrm{H}, \mathrm{Ar} \mathrm{CH}\right), 7.36$ (d, $J=8.0$ $\mathrm{Hz}, 1 \mathrm{H}, \mathrm{Ar} \mathrm{CH}), 7.47$ (s, 2H, Ar CH), 7.59 (d, $J=8.0 \mathrm{~Hz}, 1 \mathrm{H}, \mathrm{Ar} \mathrm{CH}), 7.67$ (d, $J=8.0 \mathrm{~Hz}, 2 \mathrm{H}$, Ar CH), 7.99 (s, 1H, Ar CH), 9.33 (s, 1H, NH), 11.01 (s, 1H, NH); (100 MHz, DMSO- $d_{6}$ ): $\delta$ $10.2,16.5,26.5,28.5,29.2,32.1,41.5,49.7,108.2,108.9,123.1,124.0,125.9,129.0,132.3$, 134.4, 136.8, 138.8, 143.6, 149.7, 151.8, 194.2; HRMS (ESI, TOF): calcd for $\mathrm{C}_{29} \mathrm{H}_{27} \mathrm{~N}_{4} \mathrm{O}_{2} \mathrm{Na}$ : 521.1720, Found: 521.1730.

\section{Acknowledgements}

We are thankful to Alzahra University and Iran National Science Foundation (INSF) for the financial support.

\section{References:}

1. (a) Rahmati, A.; Khalesi, Z. Tetrahedron 2012, 68, 8472-8479; (b) Vuppalapati, S. V. N.; Lee, Y. R. Tetrahedron 2012, 68, 8286-8292.

2. (a) Kusebauch, U.; Beck, B.; Messer, K.; Herdtweck, E.; Domling, A. Org. Lett. 2003, 5, 4021-4024; (b) Elinson, M. N.; Dorofeev, A. S.; Moloserdov, F. M.; Nikishin, G. I. Mol. Divers. 2009, 13, 47-52.

3. Li, Y.; Chen, H.; Shi, C.; Shi, D.; Ji, S. J. Comb. Chem. 2010, 12, 231-237.

4. (a) Hasaninejad, A.; Firoozi, S.; Mandegani, F. Tetrahedron Lett. 2013, 54, 2791-2794;

(b) Ghandi, M.; Momeni, T.; Nazeri, M. T.; Zarezadeh, N.; Kubicki, M. Tetrahedron Lett. 2013, 54, 2983-2985; (c) Jadhav, N. C.; Jagadhane, P. B.; Patile, H. V.; Telvekar, V. N. Tetrahedron Lett. 2013, 54, 3019-3021.

5. (a) Sondhi, S. M.; Dinodia, M.; Singh, J.; Rani, R. Curr. Bioact. Comp. 2007, 3, 91-108;

(b) Elguero, J.; Goya, P.; Jagerovic, N.; Silva, A. M. S. Targets Heterocycl. Systems 2002, 6, 52-98.

6. Brogden, R. N. Drugs 1986, 32, 60-70. 
7. Gursoy, A.; Demirayak, S.; Capan, G.; Erol, K.; Vural, K. Eur. J. Med. Chem. 2000, 35, 359-364.

8. Sawada, Y.; Kayakiri, H.; Abe, Y.; Mizutani, T.; Inamura, N.; Asano, M.; Hatori, C.; Aramori, I.; Oku, T.; Tanaka, H. J. Med. Chem. 2004, 47, 2853-2863.

9. Beagley, P.; Blackie, M. A. L.; Chibale, K.; Clarkson, C.; Meijboom, R.; Moss, J. R.; Smith, P.; Su, H. J. Chem. Soc. Dalton Trans. 2003, 3046-3051.

10. Ma, Z.; Hano, Y.; Nomura, T.; Chen, Y. Bioorg. Med. Chem. Lett. 2004, 14, 1193-1196.

11. Fokialakis, N.; Magiatis, P.; Chinou, L.; Mitaku, S.; Tillequin, F. Chem. Pharm. Bull. 2002, 50, 413-414.

12. Denton, T. T.; Zhang, X.; Cashman, J. R. J. Med. Chem. 2005, 48, 224-239.

13. Ryckebusch, A.; Derprez-Poulain, R.; Maes, L.; Debreu-Fontaine, M. A.; Mouray, E.; Grellier, P.; Sergheraert, C. J. Med. Chem. 2003, 46, 542-557.

14. Fossa, P.; Mosti, L.; Menozzi, G.; Marzano, C.; Baccichetti, F.; Bordin, F. Bioorg. Med. Chem. 2002, 10, 743-751.

15. Morgan, L. R.; Jursic, B. S.; Hooper, C. L.; Neumann, D. M.; Thangaraj, K.; Leblanc, B. Bioorg. Med. Chem. Lett. 2002, 12, 3407-3411.

16. Singh, R. M.; Srivastava, A. Indian J. Chem. 2005, 44B, 1868-1875.

17. (a) Krishnakumar, V.; Mandal, B. K.; Nawaz Khan, F.-R.; Jeong, E. D. Tetrahedron Lett. 2014, 55, 3717-3720; (b) Paplal, B.; Nagaraju, V.; Palakollu, S.; Kodam, K.; Sriram, V. K.; Kashinath, D. RSC Adv. 2014, 4, 54168-54174; (c) Kidwai, M.; Saxena, S.; Mohan, R. J. Heterocycl. Chem. 2005, 42, 703-705; (d) Kidwai, M.; Saxena, S.; Khalilur Rahman Khan, T. Eur. J. Med. Chem. 2005, 40, 816-819; (e) Nirav, K. S.; Manish, P. P.; Ranjan, G. P. Phosph. Sulfur Silicon Relat. Elem. 2009, 184, 2704-2719.

18. Chandra, A.; Singh, B.; Khanna, R. S.; Singh, R. J. Org. Chem. 2009, 74, 5664-5666.

19. Paul, S.; Gupta, M.; Gupta, R.; Loupy, A. Tetrahedron Lett. 2001, 42, 3827-3829.

20. (a) Ryabukhin, S. V.; Plaskon, A. S.; Volochnyuk, D. M.; Shivanyuk, A. N.; Tolmachev, A. A. Synthesis 2007, 2872-2886; (b) Siddiqui, Z. N.; Khan, K. ACS Sust. Chem. Eng. 2014, 2, 1187-1194; (c) Fu, L.; Feng, X.; Wang, J.-J.; Xun, Z.; Hu, J.-D.; Zhang, J.-J.; Zhao, Y.-W.; Huang, Z.-B.; Shi, D.-Q. ACS Comb. Sci. 2015, 17, 24-31; (d) Mirjafary, Z.; Saidian, H.; Sahandi, M.; Shojaei, L. J. Braz. Chem. Soc. 2014, 25, 1253-1260.

21. Nadaraj, V.; Selvi, S. T. J. Chem. Pharm. Res. 2012, 4, 2850-2853. 
22. Gupta, M. Bioorg. Med. Chem. Lett. 2011, 21, 4919-4923.

23. Nadaraj, V.; Selvi, S. T.; Mohan, S. J. Pharm. Res. 2009, 2, 1120-1123.

24. Fu, L.; Lin, W.; Hu, M-H.; Liu, X-C.; Huang, Z-B.; Shi, D-Q. ACS Comb. Sci. 2014, 16, $238-243$.

25. Shiri, M.; Pourabed, R.; Zadsirjan, V.; Sodagar, E.; Tetrahedron Lett. 2016, 57, 54355438.

26. (a) Shiri, M.; Farajpour, B.; Bozorgpour-Savadjani, Z.; Shintre, S. A.; Koorbanally, N. A.; Kruger, H. G.; Notash, B. Tetrahedron 2015, 71, 5531-5537; (b) Shiri, M.; Faghihi, Z.; Oskouei, H. A.; Heravi, M. M.; Fazelzadeh, S.; Notash, B. RSC Adv. 2016, 6, 92236; (c) Shiri, M.; Bozorgpour-Savadjani, Z. J. Iran. Chem. Soc. 2015, 12, 389-396; (d) Shiri, M.; Mirpour-Marzoni, Z.; Bozorgpour-Savadjani, Z.; Soleymanifard, B.; Kruger, H. G. Monatsh. Chem. 2014, 145, 1947-1952; (e) Shiri, M.; Nejatinezhad-Arani, A.; Faghihi, Z. J. Heterocycl. Chem. 2017, 54, 131-136.

27. (a) Shiri, M. Chem. Rev. 2012, 112, 3508-3549; (b) Shiri, M.; Zolfigol, M. A.; Kruger, H. G.; Tanbakouchian, Z. in Friedlander Annulation in the Synthesis of Azaheterocyclic Compounds in Advances in Heterocyclic Chemistry, ed. by A. R. Katritzky (Academic, Oxford, 2011), vol. 185, p 139; (c) Shiri, M.; Zolfigol, M. A.; Kruger, G. H.; Tanbakouchian, Z. Chem. Rev. 2010, 110, 2250-2293; (d) Soleymanifard, B.; Heravi, M. M.; Shiri, M.; Zolfigol, M. A.; Rafiee, M.; Kruger, H. G.; Naicker, T.; Rasekhmanesh, F. Tetrahedron Lett. 2012, 53, 3546; (e) Moghimi, S.; Shiri, M.; Heravi, M. M.; Kruger, H. G. Tetrahedron Lett. 2013, 54, 6215; (f) Faghihi, Z.; Oskooie, H. A.; Heravi, M. M.; Tajbakhsh, M.; Shiri, M. Monatsh. Chem. 2016, 148, 315-320.

28. Abdel-Wahab, B. F.; Khidre, R. E.; Farahat, A. A.; Sayed El-Ahl, A. A. Arkivoc 2012, (i), 211-276.

29. Wang, Z.; Ren, J.; Li, Z. Synth. Commun. 2000, 30, 763-769.

30. Dholakia, A. B.; Dholakiya, J. B.; Mehta, D. Y. Der Chem. Sinica, 2013, 4, 96.

31. Datta, B.; Madhusudana Reddy, M. B.; Pasha, M. A. Synth. Commun. 2011, 41, 23312336. 
Efficient synthesis of novel functionalized Leave this area blank for abstract info. pyrazolo-pyranoquinoline and tetrahydrodibenzo-

$[1,8]$ naphthyridinone derivatives

Morteza Shiri,* Masumeh Heydari, Vahideh Zadsirjan

Department of Chemistry, Alzahra University, Vanak, Tehran, Iran
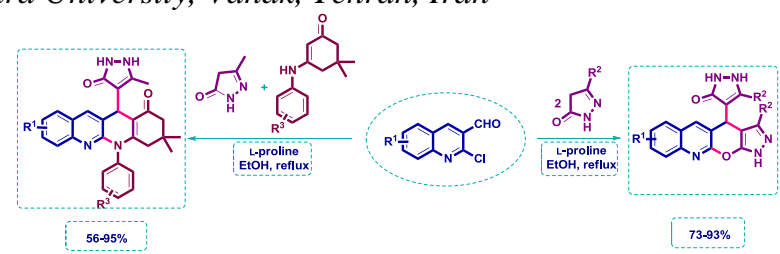